Specific Activities and DOE-STD-1027-92

Hazard Category 2 Thresholds

LANL Fact Sheet

Jim Clow

Robin DeVore

John Elder

George Heindel

William Inkret

Guthrie Miller 


\section{DISCLAIMER}

This report was prepared as an account of work sponsored by an agency of the United States Government. Neither the United States Government nor any agency thereof, nor any of their employees, make any warranty, express or implied, or assumes any legal liability or responsibility for the accuracy, completeness, or usefulness of any information, apparatus, product, or process disclosed, or represents that its use would not infringe privately owned rights. Reference herein to any specific commercial product, process, or service by trade name, trademark, manufacturer, or otherwise does not necessarily constitute or imply its endorsement, recommendation, or favoring by the United States Government or any agency thereof. The views and opinions of authors expressed herein do not necessarily state or reflect those of the United States Government or any agency thereof. 


\section{DISCLAIMER}

Portions of this document may be illegible in electronic image products. Images are produced from the best available original document. 


\title{
SPECIFIC ACTIVITIES \\ AND \\ DOE-STD-1027-92 HAZARD CATEGORY 2 THRESHOLDS \\ LANL FACT SHEET
}

by

Jim Clow, Robin DeVore, John Elder, George Heindel, William Inkret, and Guthrie Miller

\begin{abstract}
Data tables are presented to provide consistency in safety analysis work at the Los Alamos National Laboratory. Included are calculated specific activities and calculated hazard classification Category 2 threshold quantities for radionuclides listed in DOE-STD-1027-92. Some calculated threshold quantities differ from the DOE thresholds.

Calculated specific activities are also presented for plutonium material types or mixtures.
\end{abstract}

\section{Specific Activities and DOE-STD-1027-92 Hazard Category 2 Thresholds}

Purpose

To ensure more consistency in Los Alamos National

Laboratory (LANL) Safety Analysis Reports (SARs) and safety analysis work, ESH-3 will prepare and maintain LANL Fact Sheets for data used in SARs.

Scope

This fact sheet contains the following tables:

(1) Calculated specific activities (SAs)

(2) Calculated hazard classification Category 2 threshold quantities

(3) Isotopic weight fractions or percents for material mixtures 
Background

Clarification of categories

Category 3

DOE Nuclear Facility

Category 2

DOE Nuclear Facility

Category 2 threshold exceeded
Table 1 values of SA and threshold quantities were tabulated using the numbers in Table 2. Appropriate comments, information, and reference material are given in numbered notes following the tables.

LANL ESH-3 will issue Fact Sheet revisions as needed.

The threshold for hazard classification Category 2 is defined by a formula in DOE-STD-1027-92. The formula uses several specified input values and calculates a maximum plausible radionuclide quantity that will, if released, result in a 1-rem inhalation exposure to an individual at a 300 -meter distance.

A DOE facility is designated a nuclear facility if there is nuclear material present in quantities exceeding Category 3 threshold values listed in DOE-STD-1027-92 Table A.1. The quantities are small but larger than those already present in the soil or in common building materials at the site.

The threshold values for hazard classification Category 2 are larger than those for Category 3 and serve a dual purpose. They define an upper limit (maximum) of nuclear material allowed in a Category 3 facility and a threshold amount (minimum) at which the nuclear facility is designated Category 2. These threshold values have been defined in DOE-STD1027-92 by a formula that uses specified parameters chosen by DOE. Numerically, threshold values represent a plausible radionuclide quantity that, if released without mitigation of any kind, could result in a 1-rem inhalation exposure to an individual at a 300-meter distance.

A DOE facility designated as Category 2 must provide additional safety measures and systems to reduce the likelihood of an accident and provide mitigation to reduce the severity of the consequences if such an accident, even though unlikely, should occur. Also, the approved SAR for operation of a Category 2 facility generally contains a much more in-depth accident analysis than is expected for the Category 3 facility. 
Reference numbers on Tables 1 and 2 refer to Comments, Methodology, Explanations, and References on pages 8, 9, and 10 .

Table 1. Calculated Specific Activities, Category 2 Threshold Quantities, and the CEDEs Used for an Expanded List of DOE-STD-1027-92 Isotopes

\begin{tabular}{|c|c|c|c|c|c|c|c|}
\hline \multirow[b]{2}{*}{ Isotope } & \multirow[b]{2}{*}{$\begin{array}{l}\mathrm{SA}^{(2)} \\
(\mathrm{Ci} / \mathrm{gm}) \\
\end{array}$} & \multirow[b]{2}{*}{$\begin{array}{l}\text { Calculated } \\
\text { Cat-2 Th. }(5) \\
\text { (gm) }\end{array}$} & \multirow[b]{2}{*}{$\begin{array}{l}\text { 1027-A.1 } \\
\text { Cat-2 Th. } 6 \text { ) } \\
\text { (gm) }\end{array}$} & \multirow[b]{2}{*}{$\begin{array}{l}\text { Recommended } \\
\text { LANL Cat-2 } \\
\text { Threshold Values }\end{array}$} & \multicolumn{2}{|c|}{-Inhalation ${ }^{(3)}$} & \multirow[b]{2}{*}{$\begin{array}{l}\text { CEDE } \\
\text { Class-Y } \\
\text { (rem/Ci) }\end{array}$} \\
\hline & & & & & $\begin{array}{l}\text { CEDE } \\
\text { Class-D } \\
\text { (rem/Ci) }\end{array}$ & $\begin{array}{l}\text { CEDE } \\
\text { Class-W } \\
\text { (rem/Ci) }\end{array}$ & \\
\hline H-3 & $9.669 \mathrm{E}+03$ & & $3.00 \mathrm{E}+01$ & $3.00 \mathrm{E}+01^{(1)}$ & & $4.40 \mathrm{E}-03$ & \\
\hline $\mathrm{H}-3$, water & $9.669 \mathrm{E}+03$ & & $3.00 \mathrm{E}+01$ & $3.00 \mathrm{E}+01^{(1)}$ & & $6.30 \mathrm{E}+01$ & \\
\hline C-14 & $4.459 \mathrm{E}+00$ & $3.05 \mathrm{E}+05$ & $3.10 \mathrm{E}+05$ & $3.10 \mathrm{E}+05$ & & $2.10 \mathrm{E}+03$ & \\
\hline $\mathrm{Na}-22$ & $6.248 \mathrm{E}+03$ & $1.01 \mathrm{E}+00$ & $1.00 \mathrm{E}+00$ & $1.00 \mathrm{E}+00$ & $8.00 \mathrm{E}+03$ & & \\
\hline$P-32(6 b)$ & $2.857 \mathrm{E}+05$ & $1.54 \mathrm{E}-02$ & $1.50 \mathrm{E}-04$ & $1.54 \mathrm{E}-02$ & $5.50 \mathrm{E}+03$ & $1.30 \mathrm{E}+04$ & \\
\hline P-33 & $1.564 \mathrm{E}+05$ & $1.92 \mathrm{E}-01$ & $1.90 \mathrm{E}-01$ & $1.90 \mathrm{E}-01$ & $6.10 \mathrm{E}+02$ & $1.90 \mathrm{E}+03$ & \\
\hline P-32, acid $(6 b)$ & $2.857 \mathrm{E}+05$ & $7.69 \mathrm{E}+00$ & $7.70 \mathrm{E}-02$ & $7.69 \mathrm{E}+00$ & $5.50 \mathrm{E}+03$ & $1.30 \mathrm{E}+04$ & \\
\hline P-33, acid & $1.564 \mathrm{E}+05$ & $9.62 \mathrm{E}+01$ & $9.60 \mathrm{E}+01$ & $9.60 \mathrm{E}+01$ & $6.10 \mathrm{E}+02$ & $1.90 \mathrm{E}+03$ & \\
\hline S-35 & $4.268 \mathrm{E}+04$ & $5.82 \mathrm{E}-01$ & $5.80 \mathrm{E}-01$ & $5.80 \mathrm{E}-01$ & $2.90 \mathrm{E}+02$ & $2.30 \mathrm{E}+03$ & \\
\hline Cl-36 & $3.312 \mathrm{E}-02$ & $4.31 \mathrm{E}+04$ & $4.30 \mathrm{E}+04$ & $4.30 \mathrm{E}+04$ & $2.10 \mathrm{E}+03$ & $2.00 \mathrm{E}+04$ & \\
\hline $\mathrm{K}-40$ & $6.986 \mathrm{E}-06$ & $6.78 \mathrm{E}+08$ & $6.80 \mathrm{E}+08$ & $6.80 \mathrm{E}+08$ & $1.20 \mathrm{E}+04$ & & \\
\hline $\mathrm{Ca}-45$ & $1.758 \mathrm{E}+04$ & $2.66 \mathrm{E}+02$ & $2.60 \mathrm{E}+02$ & $2.60 \mathrm{E}+02$ & & $6.10 \mathrm{E}+03$ & \\
\hline $\mathrm{Ca}-47$ & $6.124 E+05$ & $7.78 \mathrm{E}+00$ & $7.80 \mathrm{E}+00$ & $7.80 \mathrm{E}+00$ & & $5.50 \mathrm{E}+03$ & \\
\hline Sc-46 & $3.387 \mathrm{E}+04$ & $4.03 E+01$ & $4.00 \mathrm{E}+01$ & $4.00 \mathrm{E}+01$ & & & $2.00 \mathrm{E}+04$ \\
\hline $\mathrm{Ti}-44$ & $1.730 \mathrm{E}+02$ & $1.86 \mathrm{E}+02$ & $1.90 \mathrm{E}+02$ & $1.90 \mathrm{E}+02$ & $4.50 \mathrm{E}+05$ & $1.70 \mathrm{E}+05$ & $8.90 \mathrm{E}+05$ \\
\hline$V-48$ & $1.703 \mathrm{E}+05$ & $1.79 \mathrm{E}+01$ & $1.80 \mathrm{E}+01$ & $1.80 \mathrm{E}+01$ & $4.60 \mathrm{E}+03$ & $8.00 \mathrm{E}+03$ & \\
\hline $\mathrm{Cr}-51$ & $9.242 E+04$ & $1.13 E+03$ & $1.10 \mathrm{E}+03$ & $1.10 \mathrm{E}+03$ & $1.10 \mathrm{E}+02$ & $2.10 \mathrm{E}+02$ & $2.60 \mathrm{E}+02$ \\
\hline$M n-52(6 c)$ & $4.494 \mathrm{E}+05$ & $8.80 \mathrm{E}+00$ & $3.90 \mathrm{E}+01$ & $8.80 \mathrm{E}+00$ & $4.50 \mathrm{E}+03$ & $5.60 \mathrm{E}+03$ & \\
\hline $\mathrm{Fe}-55$ & $2.409 \mathrm{E}+03$ & $4.56 \mathrm{E}+03$ & $4.60 \mathrm{E}+03$ & $4.60 \mathrm{E}+03$ & $2.60 E+03$ & $1.20 \mathrm{E}+03$ & \\
\hline Fe-59 & $4.962 \mathrm{E}+04$ & $3.70 \mathrm{E}+01$ & $3.70 \mathrm{E}+01$ & $3.70 \mathrm{E}+01$ & $1.50 \mathrm{E}+04$ & $9.90 \mathrm{E}+03$ & \\
\hline $\mathrm{Co}-60$ & $1.131 \mathrm{E}+03$ & $1.67 \mathrm{E}+02$ & $1.70 \mathrm{E}+02$ & $1.70 \mathrm{E}+02$ & & $3.00 \mathrm{E}+04$ & $1.50 \mathrm{E}+05$ \\
\hline $\mathrm{Ni}-63$ & $5.677 \mathrm{E}+01$ & $7.99 \mathrm{E}+04$ & $8.00 \mathrm{E}+04$ & $8.00 \mathrm{E}+04$ & & $6.30 \mathrm{E}+03$ & vapor \\
\hline $\mathrm{Zn}-65$ & $8.229 E+03$ & $1.90 \mathrm{E}+02$ & $1.90 \mathrm{E}+02$ & $1.90 \mathrm{E}+02$ & & & $1.80 \mathrm{E}+04$ \\
\hline $\mathrm{Ge}-68$ & $6.667 E+03$ & $8.75 \mathrm{E}+01$ & $8.80 \mathrm{E}+01$ & $8.80 \mathrm{E}+01$ & $1.30 \mathrm{E}+03$ & $4.90 \mathrm{E}+04$ & \\
\hline $\mathrm{Se}-75^{(6 b)}$ & $1.469 \mathrm{E}+04$ & $2.32 \mathrm{E}+01$ & $2.40 \mathrm{E}+02$ & $2.32 \mathrm{E}+01$ & $7.10 \mathrm{E}+03$ & $8.20 \mathrm{E}+03$ & \\
\hline $\mathrm{Kr}-85$ & $3.933 \mathrm{E}+02$ & $7.16 \mathrm{E}+04$ & $7.20 \mathrm{E}+04$ & $7.20 \mathrm{E}+04$ & & & \\
\hline Sr 89 & $2.905 E+04$ & $2.66 \mathrm{E}+01$ & $2.70 \mathrm{E}+01$ & $2.70 \mathrm{E}+01$ & $5.90 \mathrm{E}+03$ & & $3.70 \mathrm{E}+04$ \\
\hline Sr-90 & $1.380 \mathrm{E}+02$ & $1.59 \mathrm{E}+02$ & $1.60 \mathrm{E}+02$ & $1.60 \mathrm{E}+02$ & $2.30 \mathrm{E}+05$ & & $1.30 \mathrm{E}+06$ \\
\hline Y-91 & $2.453 \mathrm{E}+04$ & $2.65 \mathrm{E}+0 \mathrm{I}$ & $2.70 \mathrm{E}+01$ & $2.70 \mathrm{E}+01$ & & $2.90 \mathrm{E}+04$ & $4.40 \mathrm{E}+04$ \\
\hline $\mathrm{Zr}-93$ & $2.564 \mathrm{E}-03$ & $3.48 \mathrm{E}+07$ & $3.60 \mathrm{E}+07$ & $3.60 \mathrm{E}+07$ & $3.20 \mathrm{E}+05$ & $8.10 \mathrm{E}+04$ & $7.40 \mathrm{E}+04$ \\
\hline Zr-9 5 & $2.147 \mathrm{E}+04$ & $6.88 \mathrm{E}+01$ & $6.90 \mathrm{E}+01$ & $6.90 \mathrm{E}+01$ & $1.90 \mathrm{E}+04$ & $1.30 \mathrm{E}+04$ & $1.80 \mathrm{E}+0$ \\
\hline $\mathrm{Nb}-94$ & $1.903 \mathrm{E}-01$ & $4.54 \mathrm{E}+05$ & $4.60 \mathrm{E}+05$ & $4.60 \mathrm{E}+05$ & & $2.60 \mathrm{E}+04$ & $3.30 \mathrm{E}+05$ \\
\hline
\end{tabular}


Table 1-Continued

\begin{tabular}{|c|c|c|c|c|c|c|c|}
\hline \multirow[b]{2}{*}{ Isotope } & \multirow[b]{2}{*}{$\begin{array}{l}\mathrm{SA}^{(2)} \\
(\mathrm{Ci} / \mathrm{gm})\end{array}$} & \multirow[b]{2}{*}{$\begin{array}{l}\text { Calculated } \\
\text { Cat-2 Th. }{ }^{(5)} \\
(\mathrm{gm})\end{array}$} & \multirow[b]{2}{*}{$\begin{array}{l}\text { 1027-A.1 } \\
\text { Cat-2 } \\
\text { Th. }^{(6)}(\mathrm{gm})\end{array}$} & \multirow[b]{2}{*}{$\begin{array}{l}\text { Recommended } \\
\text { LANL Cat-2 } \\
\text { Threshold } \\
\text { Values } \\
\end{array}$} & \multicolumn{3}{|c|}{-..---Inhalation ${ }^{(3)}$} \\
\hline & & & & & $\mid \begin{array}{c}\text { CEDE } \\
\text { Class-D } \\
\text { (rem/Ci) }\end{array}$ & $\begin{array}{l}\text { CEDE } \\
\text { Class-W } \\
\text { (rem/Ci) }\end{array}$ & $\begin{array}{l}\text { CEDE } \\
\text { Class-Y } \\
\text { (rem/Ci) }\end{array}$ \\
\hline Mo-99 & $4.794 \mathrm{E}+05$ & $1.62 \mathrm{E}+01$ & $1.60 \mathrm{E}+01$ & $1.60 \mathrm{E}+01$ & $2.00 \mathrm{E}+03$ & & $3.60 \mathrm{E}+03$ \\
\hline Tc-99 & $1.688 \mathrm{E}-02$ & $2.26 \mathrm{E}+08$ & $2.30 \mathrm{E}+08$ & $2.30 \mathrm{E}+08$ & $8.40 \mathrm{E}+02$ & $7.50 \mathrm{E}+03$ & \\
\hline $\mathrm{Ru}-106$ & $3.356 \mathrm{E}+03$ & $1.93 \mathrm{E}+00$ & $1.90 \mathrm{E}+00$ & $1.90 \mathrm{E}+00$ & $5.70 \mathrm{E}+04$ & $9.30 \mathrm{E}+04$ & $4.40 \mathrm{E}+05$ \\
\hline $\mathrm{Ag}-110 \mathrm{~m}$ & $4.710 \mathrm{E}+03$ & $1.12 \mathrm{E}+02$ & $1.10 \mathrm{E}+02$ & $1.10 \mathrm{E}+02$ & $3.80 \mathrm{E}+04$ & $2.70 \mathrm{E}+04$ & $5.30 \mathrm{E}+04$ \\
\hline Cd-109 & $2.644 \mathrm{E}+03$ & $1.08 \mathrm{E}+02$ & $1.10 \mathrm{E}+02$ & $1.10 \mathrm{E}+02$ & $1.00 \mathrm{E}+05$ & $3.60 \mathrm{E}+04$ & $4.20 \mathrm{E}+04$ \\
\hline $\mathrm{Cd}-113$ & $3.517 \mathrm{E}-13$ & $5.08 \mathrm{E}+16$ & $5.30 \mathrm{E}+16$ & $5.30 \mathrm{E}+16$ & $1.60 \mathrm{E}+06$ & $4.70 \mathrm{E}+05$ & $3.70 \mathrm{E}+05$ \\
\hline In- $114 \mathrm{~m}$ & $2.313 \mathrm{E}+04$ & $1.58 \mathrm{E}+01$ & $1.60 \mathrm{E}+01$ & $1.60 \mathrm{E}+01$ & $7.80 \mathrm{E}+04$ & $4.90 \mathrm{E}+04$ & \\
\hline Sn-113 & $1.004 \mathrm{E}+04$ & $3.20 \mathrm{E}+02$ & $3.20 \mathrm{E}+02$ & $3.20 \mathrm{E}+02$ & $3.90 \mathrm{E}+03$ & $8.90 \mathrm{E}+03$ & \\
\hline Sn-123 & $8.229 \mathrm{E}+03$ & $1.16 \mathrm{E}+02$ & $1.20 \mathrm{E}+02$ & $1.20 \mathrm{E}+02$ & $7.90 \mathrm{E}+03$ & $3.00 \mathrm{E}+04$ & \\
\hline $\mathrm{Sn}-126$ & $2.839 \mathrm{E}-02$ & $1.17 \mathrm{E}+07$ & $1.20 \mathrm{E}+07$ & $1.20 \mathrm{E}+07$ & $8.60 \mathrm{E}+04$ & $7.40 \mathrm{E}+04$ & \\
\hline Sb-124 & $1.749 E+04$ & $7.46 \mathrm{E}+01$ & $7.50 \mathrm{E}+01$ & $7.50 \mathrm{E}+01$ & $5.50 \mathrm{E}+03$ & $2.10 \mathrm{E}+04$ & \\
\hline$S b-126^{(6 a)}$ & $8.357 \mathrm{E}+04$ & $3.03 \mathrm{E}+01$ & $3.00 \mathrm{E}+00$ & $3.03 \mathrm{E}+01$ & $4.60 \mathrm{E}+03$ & $1.00 \mathrm{E}+04$ & \\
\hline $\mathrm{Te}-127 \mathrm{~m}^{(6 a)}$ & $9.432 \mathrm{E}+03$ & $1.59 \mathrm{E}+01$ & $1.60 \mathrm{E}-01$ & $1.59 \mathrm{E}+01$ & $1.20 \mathrm{E}+04$ & $1.90 \mathrm{E}+04$ & \\
\hline $\mathrm{Te}-129 \mathrm{~m}$ & $3.021 E+04$ & $4.72 E+00$ & $4.70 \mathrm{E}+00$ & $4.70 \mathrm{E}+00$ & $8.00 \mathrm{E}+03$ & $2.00 \mathrm{E}+04$ & \\
\hline I- -125 & $1.735 \mathrm{E}+04$ & $1.37 \mathrm{E}-01$ & $1.40 \mathrm{E}-01$ & $1.40 \mathrm{E}-01$ & $2.40 \mathrm{E}+04$ & & \\
\hline I-131 & $1.240 \mathrm{E}+05$ & $1.43 \mathrm{E}-02$ & $1.40 \mathrm{E}-02$ & $1.40 \mathrm{E}-02$ & $3.20 \mathrm{E}+04$ & & \\
\hline $\mathrm{Xe}-133$ & $1.870 \mathrm{E}+05$ & $9.58 \mathrm{E}+00$ & $9.60 \mathrm{E}+00$ & $9.60 \mathrm{E}+00$ & & & \\
\hline Cs-134 & $1.294 \mathrm{E}+03$ & $4.62 \mathrm{E}+01$ & $4.60 \mathrm{E}+01$ & $4.60 \mathrm{E}+01$ & $4.70 \mathrm{E}+04$ & & \\
\hline Cs- 137 & $8.653 \mathrm{E}+01$ & $1.03 E+03$ & $1.00 \mathrm{E}+03$ & $1.00 \mathrm{E}+03$ & $3.20 \mathrm{E}+04$ & & \\
\hline $\mathrm{Ba}-133$ & $2.513 \mathrm{E}+02$ & $1.61 E+04$ & $1.60 \mathrm{E}+04$ & $1.60 \mathrm{E}+04$ & $6.90 \mathrm{E}+03$ & & \\
\hline Ba-140 & $7.292 \mathrm{E}+04$ & $1.06 \mathrm{E}+02$ & $1.10 \mathrm{E}+02$ & $1.10 \mathrm{E}+02$ & $3.60 \mathrm{E}+03$ & & \\
\hline $\mathrm{Ce}-141$ & $2.849 \mathrm{E}+04$ & $1.17 \mathrm{E}+02$ & $1.20 \mathrm{E}+02$ & $1.20 \mathrm{E}+02$ & & $7.10 \mathrm{E}+03$ & $8.50 \mathrm{E}+03$ \\
\hline Ce-144 & $3.193 E+03$ & $2.56 \mathrm{E}+01$ & $2.60 \mathrm{E}+01$ & $2.60 \mathrm{E}+01$ & & $1.90 \mathrm{E}+05$ & $3.50 \mathrm{E}+05$ \\
\hline Pm-145 & $1.394 \mathrm{E}+02$ & $7.59 \mathrm{E}+03$ & $7.60 \mathrm{E}+03$ & $7.60 \mathrm{E}+03$ & & $2.30 \mathrm{E}+04$ & $2.70 E+04$ \\
\hline Pm-147 & $9.275 \mathrm{E}+02$ & $9.06 \mathrm{E}+02$ & $8.00 \mathrm{E}+02$ & $9.06 \mathrm{E}+02$ & & $2.50 \mathrm{E}+04$ & $3.40 \mathrm{E}+04$ \\
\hline Sm-151 & $2.632 \mathrm{E}+01$ & $3.74 \mathrm{E}+04$ & $3.70 \mathrm{E}+04$ & $3.70 \mathrm{E}+04$ & & $2.90 \mathrm{E}+04$ & \\
\hline Eu-152 & $1.810 \mathrm{E}+02$ & $7.16 \mathrm{E}+02$ & $7.50 \mathrm{E}+02$ & $7.50 \mathrm{E}+02$ & & $2.20 \mathrm{E}+05$ & \\
\hline Eu-154 & $2.732 \mathrm{E}+02$ & $4.01 \mathrm{E}+02$ & $4.20 \mathrm{E}+02$ & $4.20 \mathrm{E}+02$ & $2.60 \mathrm{E}+05$ & & \\
\hline Eu-155 & $4.709 \mathrm{E}+02$ & $1.55 \mathrm{E}+03$ & $1.60 \mathrm{E}+03$ & $1.60 \mathrm{E}+03$ & & $3.90 \mathrm{E}+04$ & \\
\hline Gd-153 & $3.532 \mathrm{E}+03$ & $3.84 \mathrm{E}+02$ & $3.90 \mathrm{E}+02$ & $3.90 \mathrm{E}+02$ & $2.10 \mathrm{E}+04$ & $8.40 \mathrm{E}+03$ & \\
\hline Tb- 160 & $1.132 E+04$ & $1.12 \mathrm{E}+02$ & $1.10 \mathrm{E}+02$ & $1.10 \mathrm{E}+02$ & & $2.20 \mathrm{E}+04$ & \\
\hline Ho- $166 \mathrm{~m}$ & $1.796 \mathrm{E}+00$ & $2.21 \mathrm{E}+04$ & $2.20 \mathrm{E}+04$ & $2.20 \mathrm{E}+04$ & & $7.20 \mathrm{E}+05$ & \\
\hline $\mathrm{Tm}-170$ & $5.972 \mathrm{E}+03$ & $2.08 \mathrm{E}+02$ & $2.10 \mathrm{E}+02$ & $2.10 \mathrm{E}+02$ & & $2.30 \mathrm{E}+04$ & \\
\hline Hf- 181 & $1.701 \mathrm{E}+04$ & $1.27 \mathrm{E}+02$ & $1.30 \mathrm{E}+02$ & $1.30 \mathrm{E}+02$ & $1.30 \mathrm{E}+04$ & $1.10 \mathrm{E}+04$ & \\
\hline Ir-192 & $9.165 E+03$ & $1.33 \mathrm{E}+02$ & $1.30 \mathrm{E}+02$ & $1.30 \mathrm{E}+02$ & $1.80 \mathrm{E}+04$ & $1.30 \mathrm{E}+04$ & $2.30 \mathrm{E}+04$ \\
\hline $\mathrm{Au}-198$ & $2.446 \mathrm{E}+05$ & $3.79 \mathrm{E}+01$ & $3.80 \mathrm{E}+01$ & $3.80 \mathrm{E}+01$ & $2.90 \mathrm{E}+03$ & $2.00 \mathrm{E}+03$ & $1.80 \mathrm{E}+03$ \\
\hline
\end{tabular}


Table 1-Continued

\begin{tabular}{|c|c|c|c|c|c|c|c|}
\hline \multirow[b]{2}{*}{ Isotope } & \multirow[b]{2}{*}{$\begin{array}{l}\mathrm{SA}^{(2)} \\
(\mathrm{C} \mathrm{V} / \mathrm{gm})\end{array}$} & \multirow[b]{2}{*}{$\begin{array}{l}\text { Calculated } \\
\text { Cat-2 Th. }{ }^{(5)} \\
\text { (gm) }\end{array}$} & \multirow[b]{2}{*}{$\begin{array}{l}1027-\mathrm{A} .1 \\
\text { Cat-2 Th. }{ }^{(6)} \\
\text { (gm) }\end{array}$} & \multirow[b]{2}{*}{$\begin{array}{l}\text { Recommended } \\
\text { LANL Cat-2 } \\
\text { Threshold Values } \\
\end{array}$} & \multicolumn{3}{|c|}{-Inbalation ${ }^{(3)}$} \\
\hline & & & & & $\begin{array}{l}\text { CEDE } \\
\text { Class-D } \\
\text { (rem/Ci) }\end{array}$ & $\begin{array}{l}\text { CEDE } \\
\text { Class-W } \\
\text { (rem/Ci) }\end{array}$ & $\begin{array}{l}\text { CEDE } \\
\text { Class-Y } \\
\text { (rem/Ci) }\end{array}$ \\
\hline $\mathrm{Hg}-203(6 a)$ & $1.374 \mathrm{E}+04$ & $3.15 E+01$ & $3.10 \mathrm{E}+00$ & $3.15 \mathrm{E}+01$ & & $6.50 \mathrm{E}+03$ & \\
\hline $\mathrm{Pb}-210$ & $7.638 \mathrm{E}+01$ & $2.88 \mathrm{E}+01$ & $2.90 \mathrm{E}+01$ & $2.90 \mathrm{E}+01$ & & $1.30 \mathrm{E}+07$ & \\
\hline $\mathrm{Bi}-207^{(6 e)}$ & $5.173 \mathrm{E}+01$ & $3.75 E+04$ & $3.80 \mathrm{E}+04$ & $3.80 \mathrm{E}+04$ & $2.90 \mathrm{E}+03$ & $1.40 \mathrm{E}+04$ & \\
\hline $\mathrm{Bi}-210$ & $1.241 \mathrm{E}+05$ & $1.21 \mathrm{E}+00$ & $1.20 \mathrm{E}+00$ & $1.20 \mathrm{E}+\infty 0$ & $1.30 \mathrm{E}+04$ & $1.90 \mathrm{E}+05$ & \\
\hline Po-210 & $4.493 \mathrm{E}+03$ & $7.85 \mathrm{E}-02$ & $7.80 \mathrm{E}-02$ & $7.80 \mathrm{E}-02$ & $8.00 \mathrm{E}+06$ & $8.10 \mathrm{E}+06$ & \\
\hline $\mathrm{Rn}-222$ & $1.538 \mathrm{E}+05$ & $1.05 E+03$ & $1.10 \mathrm{E}+03$ & $1.10 \mathrm{E}+03$ & & & \\
\hline Ra-223 & $5.120 \mathrm{E}+04$ & $7.44 \mathrm{E}-02$ & $7.40 \mathrm{E}-02$ & $7.40 \mathrm{E}-02$ & & $7.50 \mathrm{E}+06$ & \\
\hline Ra-224 & $1.593 E+05$ & $6.19 \mathrm{E}-02$ & $6.10 \mathrm{E}-02$ & $6.10 \mathrm{E}-02$ & & $2.90 \mathrm{E}+06$ & \\
\hline Ra-225 & $3.921 E+04$ & $9.72 \mathrm{E}-02$ & $9.60 \mathrm{E}-02$ & $9.60 \mathrm{E}-02$ & & $7.50 \mathrm{E}+06$ & \\
\hline Ac-225 & $5.803 E+04$ & $4.92 \mathrm{E}-02$ & $4.90 \mathrm{E}-02$ & $4.90 \mathrm{E}-02$ & $1.00 \mathrm{E}+07$ & $7.50 \mathrm{E}+06$ & $8.00 \mathrm{E}+06$ \\
\hline Ac-227 & $7.237 \mathrm{E}+01$ & $5.89 \mathrm{E}-02$ & $5.90 \mathrm{E}-02$ & $5.90 \mathrm{E}-02$ & $6.70 \mathrm{E}+09$ & $1.70 \mathrm{E}+09$ & $1.20 \mathrm{E}+09$ \\
\hline Th-228 & $8.200 \mathrm{E}+02$ & $1.12 \mathrm{E}-01$ & $1.10 \mathrm{E}-01$ & $1.10 \mathrm{E}-01$ & & $2.50 \mathrm{E}+08$ & $3.10 \mathrm{E}+08$ \\
\hline Th-230 & $1.944 \mathrm{E}-02$ & $4.59 \mathrm{E}+03$ & $4.40 \mathrm{E}+03$ & $4.40 \mathrm{E}+03$ & & $3.20 \mathrm{E}+08$ & $2.60 \mathrm{E}+08$ \\
\hline Th-232 & $1.093 \mathrm{E}-07$ & $1.63 E+08$ & $1.60 \mathrm{E}+08$ & $1.60 \mathrm{E}+08$ & & $1.60 \mathrm{E}+09$ & $1.10 \mathrm{E}+09$ \\
\hline $\mathrm{U}-233^{(7)}$ & $9.642 \mathrm{E}-03$ & $2.28 \mathrm{E}+04$ & $2.30 \mathrm{E}+04$ & $2.30 \mathrm{E}+04$ & $2.70 \mathrm{E}+06$ & $7.10 \mathrm{E}+06$ & $1.30 E+08$ \\
\hline U-234 & $6.239 \mathrm{E}-03$ & $3.52 \mathrm{E}+04$ & $3.50 \mathrm{E}+04$ & $3.50 \mathrm{E}+04$ & $2.70 \mathrm{E}+06$ & $7.10 \mathrm{E}+06$ & $1.30 \mathrm{E}+08$ \\
\hline U-235(7) & $2.163 \mathrm{E}-06$ & $1.10 \mathrm{E}+08$ & $1.10 \mathrm{E}+08$ & $1.10 \mathrm{E}+08$ & $2.50 \mathrm{E}+06$ & $6.70 \mathrm{E}+06$ & $1.20 \mathrm{E}+08$ \\
\hline U-238 & $3.364 \mathrm{E}-07$ & $7.08 \mathrm{E}+08$ & $7.10 \mathrm{E}+08$ & $7.10 \mathrm{E}+08$ & $2.40 \mathrm{E}+06$ & $6.20 \mathrm{E}+06$ & $1.20 \mathrm{E}+08$ \\
\hline $\mathrm{Np}-237$ & $7.052 \mathrm{E}-04$ & 8.27E+04 & $8.30 \mathrm{E}+04$ & $8.30 \mathrm{E}+04$ & & $4.90 \mathrm{E}+08$ & \\
\hline$N p-238$ & $2.591 E+05$ & $3.53 \mathrm{E}+00$ & $3.50 \mathrm{E}+00$ & $3.50 \mathrm{E}+\infty 0$ & & $3.10 \mathrm{E}+04$ & \\
\hline Pu-238 & $1.713 E+01$ & $3.63 E+\infty 0$ & $3.60 \mathrm{E}+00$ & $3.60 \mathrm{E}+00$ & & $4.60 \mathrm{E}+08$ & $3.00 \mathrm{E}+08$ \\
\hline $\mathrm{Pu}-239(7)$ & $6.133 \mathrm{E}-02$ & $9.13 E+02$ & $9.00 \mathrm{E}+02$ & $9.00 \mathrm{E}+02$ & & $5.10 \mathrm{E}+08$ & $3.30 \mathrm{E}+08$ \\
\hline $\mathrm{Pu}-240^{(6 d)}$ & $2.268 \mathrm{E}-01$ & $2.47 \mathrm{E}+02$ & & $2.47 \mathrm{E}+02$ & & $5.10 \mathrm{E}+08$ & $3.30 \mathrm{E}+08$ \\
\hline $\mathrm{Pu}-241$ & $1.031 \mathrm{E}+02$ & $2.77 \mathrm{E}+01$ & $2.80 \mathrm{E}+01$ & $2.80 \mathrm{E}+01$ & & $1.00 \mathrm{E}+07$ & $5.70 \mathrm{E}+06$ \\
\hline $\mathrm{Pu}-242^{(6 d)}$ & $3.931 \mathrm{E}-03$ & $1.51 \mathrm{E}+04$ & & $1.51 \mathrm{E}+04$ & & $4.80 \mathrm{E}+08$ & $3.10 \mathrm{E}+08$ \\
\hline Am-241 & $3.428 \mathrm{E}+00$ & $1.60 \mathrm{E}+01$ & $1.60 \mathrm{E}+01$ & $1.60 \mathrm{E}+01$ & & $5.20 \mathrm{E}+08$ & \\
\hline Am-242m & $9.724 \mathrm{E}+00$ & $5.76 \mathrm{E}+00$ & $5.80 \mathrm{E}+00$ & $5.80 \mathrm{E}+00$ & & $5.10 \mathrm{E}+08$ & \\
\hline Am-243 & $1.997 \mathrm{E}-01$ & $2.75 E+02$ & $2.80 \mathrm{E}+02$ & $2.80 \mathrm{E}+02$ & & $5.20 \mathrm{E}+08$ & \\
\hline $\mathrm{Cm}-242$ & $3.314 \mathrm{E}+03$ & $5.07 \mathrm{E}-01$ & $5.10 \mathrm{E}-01$ & $5.10 \mathrm{E}-01$ & & $1.70 \mathrm{E}+07$ & \\
\hline $\mathrm{Cm}-245$ & $1.718 \mathrm{E}-01$ & $3.08 \mathrm{E}+02$ & 3.10E +02 & $3.10 \mathrm{E}+02$ & & $5.40 \mathrm{E}+08$ & \\
\hline Cf-252 & $5.376 \mathrm{E}+02$ & $4.09 \mathrm{E}-01$ & $7.00 \mathrm{E}-01$ & 4.09E-01 & & $1.20 \mathrm{E}+08$ & $1.30 \mathrm{E}+08$ \\
\hline
\end{tabular}


Table 2. Half-Lives, CSDEs, and Other Numerical Values Used for Calculations

\begin{tabular}{|c|c|c|c|c|c|}
\hline Isotope & $\begin{array}{l}\text { Half-life }(2) \\
(y r)\end{array}$ & $\begin{array}{l}\operatorname{CSDE}(4) \\
\left(\mathrm{rem} \times \mathrm{m}^{3} \mathrm{y}\right. \\
(\mathrm{Ci} \times \mathrm{s})\end{array}$ & $\begin{array}{l}\text { RF }{ }^{(5)} \text { Specified By } \\
\text { DOE-STD-1027-92 }\end{array}$ & $\begin{array}{l}\text { Dose Factor (4) } \\
\text { (DOE/EH-0070) } \\
(\mathrm{mrem} / \mathrm{yr})\left(\text { micro } \mathrm{Ci} / \mathrm{m}^{3}\right)\end{array}$ & $\begin{array}{l}\text { Atomic } \\
\text { Weight }\end{array}$ \\
\hline H-3 & $1.233 E+01$ & $0.00 \mathrm{E}+00$ & $1.000 \mathrm{E}+00$ & $0.00 \mathrm{E}+00$ & 3 \\
\hline H-3, water & 1.233E+01 & $0.00 \mathrm{E}+00$ & $1.000 \mathrm{E}-03$ & $0.00 \mathrm{E}+00$ & 3 \\
\hline C-14 & $5.730 E+03$ & $0.00 \mathrm{E}+00$ & $1.000 \mathrm{E}-02$ & $0.00 \mathrm{E}+00$ & 14 \\
\hline $\mathrm{Na}-22$ & $2.602 \mathrm{E}+00$ & $3.55 \mathrm{E}-01$ & $5.000 \mathrm{E}-01$ & $1.12 E+04$ & 22 \\
\hline$P-32^{(6 b)}$ & $3.912 \mathrm{E}-02$ & $0.00 \mathrm{E}+00$ & $5.000 \mathrm{E}-01$ & $0.00 \mathrm{E}+00$ & 32 \\
\hline P-33 & $6.931 \mathrm{E}-02$ & $0.00 \mathrm{E}+00$ & $5.000 \mathrm{E}-01$ & $0.00 \mathrm{E}+00$ & 33 \\
\hline P-32, acid $(6 b)$ & $3.912 \mathrm{E}-02$ & $0.00 \mathrm{E}+00$ & $1.000 \mathrm{E}-03$ & $0.00 \mathrm{E}+00$ & 32 \\
\hline P-33, acid & $6.931 \mathrm{E}-02$ & $0.00 \mathrm{E}+00$ & $1.000 \mathrm{E}-03$ & $0.00 \mathrm{E}+00$ & 33 \\
\hline S-35 & $2.394 \mathrm{E}-01$ & $0.00 \mathrm{E}+00$ & $5.000 \mathrm{E}-01$ & $0.00 \mathrm{E}+00$ & 35 \\
\hline $\mathrm{Cl}-36$ & $3.000 \mathrm{E}+05$ & $1.34 \mathrm{E}-09$ & $1.000 \mathrm{E}+00$ & $4.24 \mathrm{E}-05$ & 36 \\
\hline$K-40$ & $1.280 \mathrm{E}+09$ & $2.57 \mathrm{E}-02$ & $5.000 \mathrm{E}-01$ & $8.11 E+02$ & 40 \\
\hline $\mathrm{Ca}-45$ & $4.520 \mathrm{E}-01$ & $2.59 \mathrm{E}-12$ & $1.000 \mathrm{E}-03$ & $8.17 E-08$ & 45 \\
\hline $\mathrm{Ca}-47$ & $1.243 \mathrm{E}-02$ & $1.74 \mathrm{E}-01$ & $1.000 \mathrm{E}-03$ & $5.49 \mathrm{E}+03$ & 47 \\
\hline Sc-46 & $2.296 \mathrm{E}-01$ & 3.33E-01 & $1.000 \mathrm{E}-03$ & $1.05 E+04$ & 46 \\
\hline $\mathrm{Ti}-44$ & $4.700 \mathrm{E}+01$ & 2.13E-02 & $1.000 \mathrm{E}-03$ & $6.73 E+02$ & 44 \\
\hline$V-48$ & $4.376 \mathrm{E}-02$ & 4.79E-01 & $1.000 \mathrm{E}-03$ & $1.51 E+04$ & 48 \\
\hline Cr-51 & $7.588 \mathrm{E}-02$ & $5.01 \mathrm{E}-03$ & $1.000 \mathrm{E}-03$ & $1.58 \mathrm{E}+02$ & 51 \\
\hline $\mathrm{Mn}-52$ & $1.531 \mathrm{E}-02$ & $5.68 \mathrm{E}-01$ & $1.000 \mathrm{E}-03$ & $1.79 \mathrm{E}+04$ & 52 \\
\hline Fe-55 & $2.700 \mathrm{E}+00$ & 3.65E-06 & $1.000 \mathrm{E}-03$ & $1.15 \mathrm{E}-01$ & 55 \\
\hline Fe-59 & $1.222 \mathrm{E}-01$ & $1.96 \mathrm{E}-01$ & $1.000 \mathrm{E}-03$ & $6.17 \mathrm{E}+03$ & 59 \\
\hline Co-60 & $5.271 \mathrm{E}+00$ & $4.12 \mathrm{E}-01$ & $1.000 \mathrm{E}-03$ & $1.30 \mathrm{E}+04$ & 60 \\
\hline $\mathrm{Ni}-63$ & $1.000 \mathrm{E}+02$ & $0.00 \mathrm{E}+00$ & $1.000 \mathrm{E}-03$ & $0.00 \mathrm{E}+00$ & 63 \\
\hline $\mathrm{Zn}-65$ & $6.687 \mathrm{E}-01$ & $9.58 \mathrm{E}-02$ & $1.000 \mathrm{E}-03$ & $3.02 E+03$ & 65 \\
\hline Ge-68 & $7.889 \mathrm{E}-01$ & 1.43E-05 & $1.000 \mathrm{E}-03$ & $4.52 E-01$ & 68 \\
\hline $\mathrm{Se}-75^{(6 b)}$ & $3.246 \mathrm{E}-01$ & $6.28 \mathrm{E}-02$ & $1.000 \mathrm{E}-02$ & $1.98 \mathrm{E}+03$ & 75 \\
\hline $\mathrm{Kr}-85$ & $1.070 \mathrm{E}+01$ & 3.55E-04 & $1.000 \mathrm{E}+00$ & $1.12 \mathrm{E}+01$ & 85 \\
\hline Sr-89 & $1.383 \mathrm{E}-01$ & $2.27 \mathrm{E}-05$ & $1.000 \mathrm{E}-03$ & $7.17 \mathrm{E}-01$ & 89 \\
\hline Sr-90 & $2.880 \mathrm{E}+01$ & $0.00 \mathrm{E}+00$ & $1.000 \mathrm{E}-03$ & $0.00 \mathrm{E}+00$ & 90 \\
\hline Y-91 & $1.603 \mathrm{E}-01$ & $5.93 \mathrm{E}-04$ & $1.000 \mathrm{E}-03$ & $1.87 \mathrm{E}+01$ & 91 \\
\hline $\mathrm{Zr}-93$ & $1.500 E+06$ & $0.00 E+00$ & $1.000 \mathrm{E}-03$ & $0.00 E+00$ & 93 \\
\hline Zr-9 5 & 1.753E-01 & $1.21 \mathrm{E}-01$ & $1.000 \mathrm{E}-03$ & $3.81 \mathrm{E}+03$ & 95 \\
\hline $\mathrm{Nb}-94$ & $2.000 \mathrm{E}+04$ & $2.60 \mathrm{E}-01$ & $1.000 \mathrm{E}-03$ & $8.20 E+03$ & 94 \\
\hline Mo-99 & $7.536 \mathrm{E}-03$ & $2.53 \mathrm{E}-02$ & $1.000 \mathrm{E}-03$ & $7.99 \mathrm{E}+02$ & 99 \\
\hline Tc-99 & $2.140 \mathrm{E}+05$ & 8.40E-08 & $1.000 \mathrm{E}-03$ & $2.65 \mathrm{E}-03$ & 99 \\
\hline Ru-106 & $1.005 \mathrm{E}+00$ & $0.00 \mathrm{E}+00$ & $1.000 \mathrm{E}-02$ & $0.00 \mathrm{E}+00$ & 106 \\
\hline $\mathrm{Ag}-110 \mathrm{~m}$ & $6.903 \mathrm{E}-01$ & $4.50 \mathrm{E}-01$ & $1.000 \mathrm{E}-03$ & $1.42 \mathrm{E}+04$ & 110 \\
\hline \multirow[t]{2}{*}{ Cd-109 } & $1.241 \mathrm{E}+00$ & $3.49 E-04$ & $1.000 \mathrm{E}-03$ & $1.10 \mathrm{E}+01$ & 109 \\
\hline & & & & \multicolumn{2}{|c|}{ Continued on next page } \\
\hline
\end{tabular}


Table 2 -Continued

\begin{tabular}{|c|c|c|c|c|c|}
\hline Isotope & $\begin{array}{l}\text { Half-life (2) } \\
(y r)\end{array}$ & $\begin{array}{l}\operatorname{CSDE}(4) \\
\left(\mathrm{rem} \times \mathrm{m}^{3}\right) \\
(\mathrm{Ci} \times \mathrm{s}) \\
\end{array}$ & $\begin{array}{l}\mathrm{RF}^{(5)} \text { Specified By } \\
\text { DOE-STD-1027-92 }\end{array}$ & $\begin{array}{l}\text { Dose Factor (4) } \\
\text { (DOE/EH-0070) } \\
(\mathrm{mrem} / \mathrm{yr}) /\left(\text { micro } \mathrm{Ci} / \mathrm{m}^{3}\right)\end{array}$ & $\begin{array}{l}\text { Atomic } \\
\text { Weight }\end{array}$ \\
\hline Cd-113 & $9.000 \mathrm{E}+15$ & $0.00 \mathrm{E}+00$ & $1.000 \mathrm{E}-03$ & $0.00 \mathrm{E}+00$ & 113 \\
\hline In-114m & $1.356 \mathrm{E}-01$ & $1.46 \mathrm{E}-02$ & $1.000 \mathrm{E}-03$ & $4.59 \mathrm{E}+02$ & 114 \\
\hline Sn-113 & 3.153E-01 & 1.33E-03 & $1.000 \mathrm{E}-03$ & $4.19 \mathrm{E}+01$ & 113 \\
\hline Sn-123 & $3.534 \mathrm{E}-01$ & $1.14 \mathrm{E}-03$ & $1.000 \mathrm{E}-03$ & $3.60 \mathrm{E}+01$ & 123 \\
\hline Sn-126 & $1.000 \mathrm{E}+05$ & 7.74E-03 & $1.000 \mathrm{E}-03$ & $2.44 \mathrm{E}+02$ & 126 \\
\hline$S b-124$ & $1.649 \mathrm{E}-01$ & $3.15 \mathrm{E}-01$ & $1.000 \mathrm{E}-03$ & $9.95 \mathrm{E}+03$ & 124 \\
\hline$S b-126^{(6 a)}$ & $3.397 \mathrm{E}-02$ & 4.47E-01 & $1.000 \mathrm{E}-03$ & $1.41 \mathrm{E}+04$ & 126 \\
\hline$T e-127 m^{(6 a)}$ & $2.986 \mathrm{E}-01$ & $4.95 \mathrm{E}-04$ & $1.000 \mathrm{E}-02$ & $1.56 \mathrm{E}+01$ & 127 \\
\hline Te-129m & 9.177E-02 & $5.45 \mathrm{E}-03$ & $1.000 \mathrm{E}-02$ & $1.72 \mathrm{E}+02$ & 129 \\
\hline I-125 & $1.649 \mathrm{E}-01$ & $1.77 \mathrm{E}-03$ & $5.000 \mathrm{E}-01$ & $5.57 \mathrm{E}+01$ & 125 \\
\hline I-131 & $2.202 \mathrm{E}-02$ & $6.06 \mathrm{E}-02$ & $5.000 \mathrm{E}-01$ & $1.91 \mathrm{E}+03$ & 131 \\
\hline$X e-133$ & $1.438 \mathrm{E}-02$ & $5.58 \mathrm{E}-03$ & $1.000 \mathrm{E}+00$ & $1.76 \mathrm{E}+02$ & 133 \\
\hline Cs-134 & $2.062 \mathrm{E}+00$ & $2.54 \mathrm{E}-01$ & $1.000 \mathrm{E}-02$ & $8.01 E+03$ & 134 \\
\hline Cs-137 & $3.017 \mathrm{E}+01$ & $0.00 \mathrm{E}+00$ & $1.000 \mathrm{E}-02$ & $0.00 \mathrm{E}+00$ & 137 \\
\hline Ba-133 & $1.070 \mathrm{E}+01$ & $5.83 \mathrm{E}-02$ & $1.000 \mathrm{E}-03$ & $1.84 \mathrm{E}+03$ & 133 \\
\hline Ba-140 & $3.504 \mathrm{E}-02$ & $2.98 \mathrm{E}-02$ & $1.000 \mathrm{E}-03$ & $9.41 \mathrm{E}+02$ & 140 \\
\hline Ce-141 & $8.903 E-02$ & $1.22 \mathrm{E}-02$ & $1.000 \mathrm{E}-03$ & $3.86 \mathrm{E}+02$ & 141 \\
\hline $\mathrm{Ce}-144$ & $7.780 \mathrm{E}-01$ & $2.88 \mathrm{E}-03$ & $1.000 \mathrm{E}-03$ & $9.09 \mathrm{E}+01$ & 144 \\
\hline Pm-145 & $1.770 \mathrm{E}+01$ & $2.65 \mathrm{E}-03$ & $1.000 \mathrm{E}-03$ & 8.37E+01 & 145 \\
\hline Pm-147 & $2.623 \mathrm{E}+00$ & $5.80 \mathrm{E}-07$ & $1.000 \mathrm{E}-03$ & $1.83 \mathrm{E}-02$ & 147 \\
\hline Sm-151 & $9.000 \mathrm{E}+01$ & 1.45E-07 & $1.000 \mathrm{E}-03$ & $4.56 \mathrm{E}-03$ & 151 \\
\hline Eu-152 & $1.300 E+01$ & $1.87 \mathrm{E}-01$ & $1.000 \mathrm{E}-03$ & $5.90 \mathrm{E}+03$ & 152 \\
\hline Eu-154 & $8.500 \mathrm{E}+00$ & $2.06 \mathrm{E}-01$ & $1.000 \mathrm{E}-03$ & $6.50 \mathrm{E}+03$ & 154 \\
\hline Eu-155 & $4.900 \mathrm{E}+00$ & $9.10 \mathrm{E}-03$ & $1.000 \mathrm{E}-03$ & $2.87 \mathrm{E}+02$ & 155 \\
\hline Gd-153 & $6.618 \mathrm{E}-01$ & $1.41 \mathrm{E}-02$ & $1.000 \mathrm{E}-03$ & $4.46 \mathrm{E}+02$ & 153 \\
\hline$T b-160$ & $1.975 \mathrm{E}-01$ & $1.78 \mathrm{E}-01$ & $1.000 \mathrm{E}-03$ & $5.61 \mathrm{E}+03$ & 160 \\
\hline Ho-166m & $1.200 \mathrm{E}+03$ & $2.60 \mathrm{E}-01$ & $1.000 \mathrm{E}-03$ & $8.21 E+03$ & 166 \\
\hline $\operatorname{Tm}-170$ & 3.523E-01 & 7.39E-04 & $1.000 \mathrm{E}-03$ & $2.33 \mathrm{E}+01$ & 170 \\
\hline Hf- 181 & $1.162 \mathrm{E}-01$ & 8.62E-02 & $1.000 \mathrm{E}-03$ & $2.72 \mathrm{E}+03$ & 181 \\
\hline Ir -192 & 2.033E-01 & $1.30 \mathrm{E}-01$ & $1.000 \mathrm{E}-03$ & $4.11 \mathrm{E}+03$ & 192 \\
\hline Au-198 & 7.385E-03 & $6.44 \mathrm{E}-02$ & $1.000 \mathrm{E}-03$ & $2.03 \mathrm{E}+03$ & 198 \\
\hline $\mathrm{Hg}-203^{(6 a)}$ & $1.282 \mathrm{E}-01$ & $3.65 \mathrm{E}-02$ & $1.000 \mathrm{E}-02$ & $1.15 \mathrm{E}+03$ & 203 \\
\hline $\mathrm{Pb}-210$ & $2.230 \mathrm{E}-01$ & $2.12 \mathrm{E}-04$ & $1.000 \mathrm{E}-03$ & $6.70 E+00$ & 210 \\
\hline $\mathrm{Bi}-207^{(6 e)}$ & $3.340 \mathrm{E}+01$ & $2.52 \mathrm{E}-01$ & $1.000 \mathrm{E}-03$ & $7.96 \mathrm{E}+03$ & 207 \\
\hline Bi-210 & $1.372 \mathrm{E}-02$ & $0.00 \mathrm{E}+00$ & $1.000 \mathrm{E}-03$ & $0.00 \mathrm{E}+00$ & 210 \\
\hline Po-210 & $3.791 \mathrm{E}-01$ & $1.41 \mathrm{E}-06$ & $1.000 \mathrm{E}-02$ & $4.44 \mathrm{E}-02$ & 210 \\
\hline Rn-222 & $1.047 \mathrm{E}-02$ & $6.18 \mathrm{E}-05$ & $1.000 \mathrm{E}+00$ & $1.95 \mathrm{E}+00$ & 222 \\
\hline
\end{tabular}

Continued on next page 
Table 2-Continued

\begin{tabular}{|c|c|c|c|c|c|}
\hline Isotope & $\begin{array}{c}\text { Half-life } \\
\qquad(\mathrm{yr})\end{array}$ & $\begin{array}{l}\operatorname{CSDE}(4) \\
\left(\mathrm{rem} \times \mathrm{m}^{3} \mathrm{y}\right. \\
(\mathrm{Ci} \times \mathrm{s}) \\
\end{array}$ & $\begin{array}{l}\text { RF }(5) \\
\text { Specified By } \\
\text { DOE-STD-1027-92 }\end{array}$ & $\begin{array}{l}\text { Dose Factor }(4) \\
(\mathrm{DOE} / \mathrm{EH}-0070) \\
(\mathrm{mrem} / \mathrm{yr}) /\left(\mathrm{micro} \mathrm{Ci} / \mathrm{m}^{3}\right)\end{array}$ & $\begin{array}{l}\text { Atomic } \\
\text { Weight }\end{array}$ \\
\hline $\mathrm{Ra}-223$ & $3.132 \mathrm{E}-02$ & $2.13 \mathrm{E}-02$ & $1.000 \mathrm{E}-03$ & $6.72 \mathrm{E}+02$ & 223 \\
\hline Ra-224 & $1.003 \mathrm{E}-02$ & $1.60 \mathrm{E}-03$ & $1.000 \mathrm{E}-03$ & $5.06 \mathrm{E}+01$ & 224 \\
\hline $\mathrm{Ra}-225$ & $4.054 \mathrm{E}-02$ & 1.08E-03 & $1.000 \mathrm{E}-03$ & $3.40 \mathrm{E}+01$ & 225 \\
\hline Ac- 225 & $2.739 \mathrm{E}-02$ & $2.15 \mathrm{E}-03$ & $1.000 \mathrm{E}-03$ & $6.79 \mathrm{E}+01$ & 225 \\
\hline Ac-227 & $2.177 \mathrm{E}+01$ & $1.99 \mathrm{E}-05$ & $1.000 \mathrm{E}-03$ & $6.29 \mathrm{E}-01$ & 227 \\
\hline Th-228 & $1.913 E+00$ & 3.14E-04 & $1.000 \mathrm{E}-03$ & $9.89 \mathrm{E}+00$ & 228 \\
\hline Th-230 & $8.000 \mathrm{E}+04$ & $6.21 \mathrm{E}-05$ & $1.000 \mathrm{E}-03$ & $1.96 \mathrm{E}+00$ & 230 \\
\hline Th-232 & $1.410 E+10$ & $2.96 \mathrm{E}-05$ & $1.000 \mathrm{E}-03$ & $9.33 \mathrm{E}-01$ & 232 \\
\hline $\mathrm{U}-233^{(7)}$ & $1.592 \mathrm{E}+05$ & $3.80 \mathrm{E}-05$ & $1.000 \mathrm{E}-03$ & $1.20 \mathrm{E}+00$ & 233 \\
\hline U-234 & $2.450 \mathrm{E}+05$ & $2.43 \mathrm{E}-05$ & $1.000 \mathrm{E}-03$ & 7.65E-01 & 234 \\
\hline U-235 $(7)$ & $7.038 \mathrm{E}+08$ & $2.44 E-02$ & $1.000 \mathrm{E}-03$ & $7.70 \mathrm{E}+02$ & 235 \\
\hline U-238 & $4.468 \mathrm{E}+09$ & $1.65 \mathrm{E}-05$ & $1.000 \mathrm{E}-03$ & $5.19 \mathrm{E}-01$ & 238 \\
\hline$N p-237$ & $2.140 \mathrm{E}+06$ & 3.65E-03 & $1.000 \mathrm{E}-03$ & $1.15 \mathrm{E}+02$ & 237 \\
\hline Np-238 & $5.799 \mathrm{E}-03$ & $9.16 \mathrm{E}-02$ & $1.000 \mathrm{E}-03$ & $2.89 E+03$ & 238 \\
\hline Pu-238 & $8.774 \mathrm{E}+01$ & $1.40 \mathrm{E}-0.5$ & $1.000 \mathrm{E}-03$ & $4.41 \mathrm{E}-01$ & 238 \\
\hline $\mathrm{Pu}-239(7)$ & $2.440 E+04$ & $1.30 \mathrm{E}-05$ & $1.000 \mathrm{E}-03$ & $4.11 \mathrm{E}-01$ & 239 \\
\hline $\mathrm{Pu}-240^{(6 d)}$ & $6.570 \mathrm{E}+03$ & 1.37E-05 & $1.000 \mathrm{E}-03$ & 4.32E-01 & 240 \\
\hline $\mathrm{Pu}-241$ & $1.440 \mathrm{E}+01$ & $0.00 \mathrm{E}+00$ & $1.000 \mathrm{E}-03$ & $0.00 \mathrm{E}+00$ & 241 \\
\hline $\mathrm{Pu}-242^{(6 d)}$ & $3.760 \mathrm{E}+05$ & $1.16 \mathrm{E}-05$ & $1.000 \mathrm{E}-03$ & $3.67 \mathrm{E}-01$ & 242 \\
\hline Am-241 & $4.330 \mathrm{E}+02$ & $3.01 \mathrm{E}-03$ & $1.000 \mathrm{E}-03$ & $9.50 \mathrm{E}+01$ & 241 \\
\hline Am-242m & $1.520 \mathrm{E}+02$ & $7.55 \mathrm{E}-05$ & $1.000 \mathrm{E}-03$ & $2.38 E+00$ & 242 \\
\hline Am-243 & $7.370 \mathrm{E}+03$ & $8.12 \mathrm{E}-03$ & $1.000 \mathrm{E}-03$ & $2.56 \mathrm{E}+02$ & 243 \\
\hline $\mathrm{Cm}-242$ & $4.460 \mathrm{E}-01$ & $1.55 \mathrm{E}-05$ & $1.000 \mathrm{E}-03$ & $4.90 \mathrm{E}-01$ & 242 \\
\hline $\mathrm{Cm}-245$ & $8.500 \mathrm{E}+03$ & $1.13 \mathrm{E}-02$ & $1.000 \mathrm{E}-03$ & $3.57 \mathrm{E}+02$ & 245 \\
\hline Cf- 252 & $2.640 \mathrm{E}+00$ & $1.19 \mathrm{E}-05$ & $1.000 \mathrm{E}-03$ & $3.75 \mathrm{E}-01$ & 252 \\
\hline
\end{tabular}

\section{Comments, Methodology, Explanations, and References}

1. The tritium Category 2 threshold given in DOE-STD-1027-92 Table A.1 is not a calculated quantity; DOE set the value recommended by a special committee.

2. In Table 2, LANL calculated the SAs in curies per gram directly from the half-life and atomic weight using the following formula:

$\mathrm{T}_{12}=$ half-life $(\mathrm{yr})$

$\mathrm{AW}=$ atomic weight of the isotope 


$$
\begin{aligned}
\mathrm{N}_{\mathrm{o}}= & 6.023 \times 10^{23}=\text { Avagadro's number } \\
& \mathrm{SA}(\mathrm{Bq} / \mathrm{gm})=\left(0.693 \times \mathrm{N}_{\mathrm{o}}\right) /\left(\mathrm{AW} \times \mathrm{T}_{12} \times 3.154 \times 10^{7} \mathrm{~s} / \mathrm{yr}\right) \text { or, } \\
& \mathrm{SA}(\mathrm{Ci} / \mathrm{gm})=\mathrm{SA}(\mathrm{Bq} / \mathrm{gm}) /\left(3.7 \times 10^{10} \mathrm{~Bq} / \mathrm{Ci}\right) .
\end{aligned}
$$

The half-life values in Table 2 were obtained from Table of Isotopes, Seventh Edition, C. Michael Lederer and Virginia S. Shirley, Eds. (A Wiley-Interscience Publication, John Wiley \& Sons, Inc., New York, 1978).

3. The 50-year committed effective dose equivalents (CEDEs) in Table 1 were obtained from DOE/EH-0071, "Internal Dose Conversion Factors for Calculation of Dose to the Public," (July 1988).

In DOE-STD-1027-92 Table A.1, DOE apparently used the largest values of the Inhalation Class $\mathrm{D}, \mathrm{W}$, or $\mathrm{Y}$ in the interest of conservatism in calculating their Category 2 thresholds. To reproduce their results, we have done the same for this document.

4. Values for calculating the Cloud Shine Dose Equivalent (CSDE) for external exposure from a contaminated cloud of airborne particulates were obtained from DOE/EH-0070, "External Dose-Rate Conversion Factors for Calculation of Dose to the Public," (July 1988).

Table 2 contains dose factors for all isotopes. Multiplication of these Dose Factors by 3.171E-05 (rems $\times$ microcuries $\times \mathrm{yr} / \mathrm{Ci} \times \mathrm{mrem} \times \mathrm{s}$ ) converts the units and gives the CSDEs in rems $\times \mathrm{m}^{3} / \mathrm{Ci} \times \mathrm{s}$, which are also tabulated in Table 2 .

5. DOE-STD-1027-92 Attachment 1 specifies a formula and numerical values to be used when calculating hazard Category 2 thresholds. The formula is

$$
\mathrm{Q}(\text { grams })=1(\mathrm{rems}) /\{\mathrm{RF} \times \mathrm{SA} \times(\mathrm{X} / \mathrm{Q}) \times[\mathrm{CEDE} \times \mathrm{RR}+\mathrm{CSDE}]\},
$$

where

$$
\begin{aligned}
& \mathrm{SA}=(\mathrm{Ci} / \mathrm{gm}) \\
& (\mathrm{X} / \mathrm{Q})=\text { Atmospheric Dispersion Factor }\left(\mathrm{s} / \mathrm{m}^{3}\right)=1.0 \mathrm{E}-04 \text { for all isotopes } \\
& \text { (cf. DOE-STD-1027 for DOE's discussion of why this is a suitably } \\
& \text { conservative value to use) } \\
& \mathrm{RR}=\text { Respiration Rate: } 3.5 \mathrm{E}-4 \mathrm{~m}^{3} / \mathrm{s}
\end{aligned}
$$

$\mathrm{CSDE}=$ Cloud Shine Dose Equivalent. The units are: $\mathrm{rem} \times \mathrm{m}^{3} / \mathrm{Ci} \times \mathrm{s}$.

$\mathrm{RF}=$ Release Fraction: DOE-STD-1027-92 uses four different numerical values as follows:

- 1.0 for gases such as tritium, the noble gases, chlorine, etc.

- 0.5 for phosphorus, sulfur, potassium, iodine, sodium, and bromine

- 0.01 for selenium, mercury, cesium, polonium, tellurium ruthenium, and carbon

- 0.001 for other solids, powders, and liquids not listed above

This classification for the RF is based on classes of materials adopted by NRC. DOE reduced the number of classes and built in some additional conservatism (cf. DOESTD-1027-92 Attachment 1). Table 2 contains the RF for each isotope in Table 1. 
6. The hazard Category 2 thresholds from DOE-STD-1027-92 Table A.1 are tabulated in our Table 1, column 4. For DOE values that disagree with calculated values, the following comments are in order.

a. Consider Sb-126, $\mathrm{Te}-127 \mathrm{~m}$, and $\mathrm{Hg}-203$. Hazard Category 2 thresholds are given in DOE-STD-1027-92 Table A.1 both in curies and grams. Dividing the two numbers gives the SA that DOE used. For these three materials, the SA obtained by DOE's method differs from the value of SA calculated from half-life data by almost 10 times. The spread for published half-life data, however, is only a few percent. Therefore, one of the two threshold values given in DOE-STD-1027-92 Table A.1 is probably in error. The authors of this document suspect that the Category 2 gram-thresholds in DOE-STD-1027-92 Table A.1 for these three materials contains an exponential clerical error.

b. For P-32 and Se-75, the SA that DOE used agrees with our half-life calculated SA. However, our calculated threshold values and the DOE values differ by about a factor of 100 and of 10 respectively.

c. Also, significant disagreement (not factors of 10) exists for Pm-147, Mn-52, and CF-252.

d. For LANL use, we have added the isotopes Pu-240 and Pu-242 to the DOE-STD1027-92 Table A.1 list. DOE-STD-1027-92 Table A.1 does not contain Category 2 threshold values for Pu-240 and Pu-242, which we need for mixture calculations.

e. The half-life of $\mathrm{Bi}-207$ is $38 \mathrm{yr}$ according to the most current reference cited earlier (Table of Isotoptes). However, the Radiological Health Handbook, U.S. , (Department of Health Education and Welfare, Rockville, MD, 1970) and DOE/EH-0070, "External Dose-Rate Conversion Factors for Calculation of Dose to the Public," (July 1988) both give half-lives that are significantly lower, (e.g., 28-33.4 yr). The 33.4-yr half-life value leads to a calculated threshold value that agrees with DOE-STD-1027-92 Table A.1. The most recent value of $38 \mathrm{yr}$ leads to a Category 2 threshold value of $4.27 \mathrm{E}+04 \mathrm{gm}$-about $12.4 \%$ larger than the DOE-STD-1027-92 Table A.1 value of 3.80E+04.

In summary:

We believe our calculated values of the Category 2 threshold to be correct. The "Recommended Category 2 Threshold" values in Table1, Column 5 are from two sources.: (1) P-32, Mn-52, Se-75, Sb-126, Te-127m, Pm-147, Hg-203, Pu240, $P u-242$, and $C f-252$ are LANL-calculated values. (2) All others are DOE values that come within a percent or two of the LANL-calculated values.

7. Criticality considerations further restrict the calculated threshold quantities for some materials. DOE-STD-1027-92 Table A.1 gives values. This Fact Sheet gives total facility threshold values-configured so that criticality is not possible. For example, for pure Pu-239, the criticality limit is $450 \mathrm{gm}$. The Category 2 threshold limit of $900 \mathrm{gm}$ given here and in DOE-STD-1027-92 Table A.1. means that a Category 3 facility could have two separated batches of less than 450 grams each. 


\section{Mass Fractions, Specific Activities, and Specific Doses for Mixtures and Material Types}

Scope

A number of LANL facilities process, store, or utilize plutonium and uranium mixtures that contain various isotopes. This section provides recommended numbers and calculations for these mixtures. NMT-4 currently recommends the weight-percent values listed in Tables $3 \mathrm{a}$ and $3 \mathrm{~b}$. (cf. Joseph R. Wachter, "Correction to Memorandum," Los Alamos National Laboratory memorandum NMT-04-93-536 November 23, 1993).

Table 3. Recommended Specific Activities, Specific Doses, and Mass Fractions for Individual Plutonium Isotopes and Plutonium Material Types or Mixtures

Table 3a. Data for Individual Plutonium Isotopes

\begin{tabular}{cccc}
\hline Isotope & $\begin{array}{c}\mathrm{SA}(\mathrm{Ci} / \mathrm{g}) \\
(\mathrm{Ci} / \mathrm{gm})\end{array}$ & $\begin{array}{c}\mathrm{CEDE} \\
(\mathrm{rem} / \mathrm{Ci})\end{array}$ & $\begin{array}{c}\text { Specific Dose } \\
(\mathrm{rem} / \mathrm{gm})\end{array}$ \\
\hline $\mathrm{Pu}-238$ & $1.713 \mathrm{E}+01$ & $4.60 \mathrm{E}+08$ & $7.88 \mathrm{E}+09$ \\
$\mathrm{Pu}-239$ & $6.133 \mathrm{E}-02$ & $5.10 \mathrm{E}+08$ & $3.13 \mathrm{E}+07$ \\
$\mathrm{Pu}-240$ & $2.268 \mathrm{E}-01$ & $5.10 \mathrm{E}+08$ & $1.16 \mathrm{E}+08$ \\
$\mathrm{Pu}-241$ & $1.031 \mathrm{E}+02$ & $1.00 \mathrm{E}+07$ & $1.03 \mathrm{E}+09$ \\
$\mathrm{Pu}-242$ & $3.931 \mathrm{E}-03$ & $4.80 \mathrm{E}+08$ & $1.89 \mathrm{E}+06$ \\
$\mathrm{Am}-241$ & $3.428 \mathrm{E}+00$ & $5.20 \mathrm{E}+08$ & $1.78 \mathrm{E}+09$ \\
& & & \\
\hline
\end{tabular}

Table 3b. Data for Plutonium Material Types or Mixtures

\begin{tabular}{|c|c|c|c|c|c|c|c|c|c|}
\hline $\begin{array}{c}\text { Material } \\
\text { Type }\end{array}$ & $\begin{array}{c}\text { Pu-238 } \\
\text { (weight } \\
\% \text { ) }\end{array}$ & $\begin{array}{c}\text { Pu-239 } \\
\text { (weight } \\
\% \text { ) }\end{array}$ & $\begin{array}{c}\text { Pu-240 } \\
\text { (weight } \\
\% \text { ) }\end{array}$ & $\begin{array}{c}\text { Pu-241 } \\
\text { (weight \%) }\end{array}$ & $\begin{array}{l}\mathrm{Pu}-242 \\
\text { (weight \%) }\end{array}$ & $\begin{array}{c}\text { Pu-244 } \\
\text { (weight } \\
\% \text { ) }\end{array}$ & $\begin{array}{l}\text { Percent } \\
\text { (Total) }\end{array}$ & $\begin{array}{l}\text { Specific } \\
\text { Activity } \\
(\mathrm{Ci} / \mathrm{gm})\end{array}$ & $\begin{array}{c}\text { Specific Dose } \\
(\mathrm{rem} / \mathrm{gm})\end{array}$ \\
\hline MT42 $84 \%$ & 1.02 & 1.37 & 10.32 & 3.13 & 84.14 & 0.02 & 100 & 3.428136 & $1.27 \mathrm{E}+08$ \\
\hline MT42 $90 \%$ & 0.72 & 1.26 & 6.4 & 1.86 & 89.77 & & 100.01 & 2.059113 & $8.54 \mathrm{E}+07$ \\
\hline MT42 95\% & 0.45 & 0.56 & 2.47 & 0.906 & 95.58 & 0.029 & 99.995 & 1.020531 & $4.96 \mathrm{E}+07$ \\
\hline MT51 & 0.006 & 96.77 & 3.13 & 0.076 & 0.018 & & 100 & 0.145808 & $3.51 \mathrm{E}+07$ \\
\hline MT52 & 0.01 & 93.78 & 6 & 0.2 & 0.02 & & 100.01 & 0.278968 & $3.91 \mathrm{E}+07$ \\
\hline MT53 & 0.03 & 91.08 & 8.45 & 0.366 & 0.071 & & 99.997 & 0.457382 & $4.44 E+07$ \\
\hline MT54 & 0.046 & 87.42 & 11.5 & 0.81 & 0.22 & & 99.996 & 0.922401 & $5.26 \mathrm{E}+07$ \\
\hline MT55 & 0.06 & 83.88 & 14.73 & 1.03 & 0.304 & & 100.004 & 1.156697 & $5.86 \mathrm{E}+07$ \\
\hline MT56 & 0.061 & 81.9 & 16.51 & 1.18 & 0.355 & & 100.006 & 1.314288 & $6.17 \mathrm{E}+07$ \\
\hline MT57 & 0.433 & 74.63 & 20.7 & 2.55 & 1.69 & & 100.003 & 2.795066 & $1.08 \mathrm{E}+08$ \\
\hline MT83 83\% & 83.89 & 13.8 & 1.9 & 0.32 & 0.09 & & 100 & 14.7113 & $6.62 \mathrm{E}+09$ \\
\hline MT83 89\% & 89.26 & 10.07 & 0.633 & 0.021 & 0.015 & & 99.999 & 15.31775 & $7.04 \mathrm{E}+09$ \\
\hline
\end{tabular}


Dominant isotope method for initial hazard categorization

Sum-of-fractions method for final hazard categorization

Time-dependent change of Category 2 thresholds
For purposes of initial hazard categorization to determine hazard Category 2 quantities, DOE recommends using the dominant isotope Category 2 threshold value. For example, use $900 \mathrm{gm}$ for the $\mathrm{Pu}-239$-dominant MT52 and $3.6 \mathrm{gm}$ for the $\mathrm{Pu}-238$-dominant MT83. The other trace isotopes are ignored.

DOE-STD-1027-92 Attachment 1 specifies that a sum-offractions method be used for mixtures as follows. Divide the weight percent (Table 3) of each isotope in the mixture by its individual Category 2 isotopic threshold value. When the ratios are added together, the sum must be less than one. In effect, less than the maximum allowable amount of one or several isotopes means that the potential exposure (in rems) for the combined isotopes will be the same as the value that has been established as acceptable for any one of them (i.e., less than $1 \mathrm{rem}$ at a 300-meter distance).

We have calculated separately that the sum-of-fractions method generally reduces the allowed gram loading for the $\mathrm{Pu}-239$ dominant mixtures and allows increased amounts for the heatsource mixtures such as MT83.

Remember, when calculating the Category 2 threshold value for mixtures and comparing this value to the initial hazard categorization threshold quantity (for the dominant isotope), that the presence of small amounts of Am-241 will vary with time as $\mathrm{Pu}-241$ decays and can cause a time-dependent change of the threshold values. This reduction with age for the Category 2 threshold values is particularly significant only for mixtures that are 20 or more years old. Additionally, the Am-241 content data for the mixtures may be lacking. If the Am-241 content data are available, ESH-3 can calculate, as needed, the reduced threshold values caused by aging. However, through methodology and parameter choices, DOE has built considerable conservatism into their threshold values. Therefore, calculating time-dependent threshold values is probably unnecessary. 